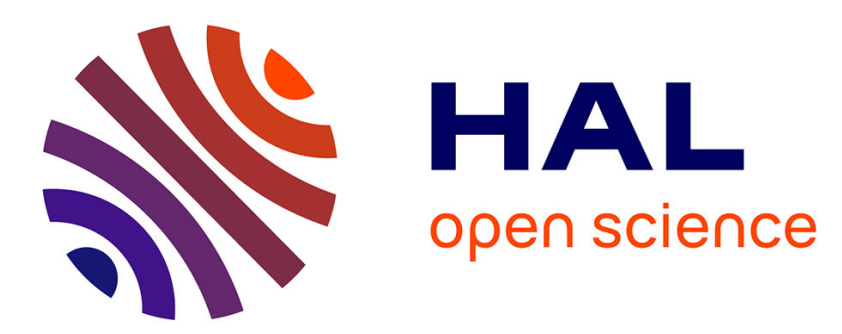

\title{
Study of G-Protein Coupled Receptor Signaling in Membrane Environment by Plasmon Waveguide Resonance
}

\author{
Isabel D. Alves, Sophie Lecomte
}

\section{- To cite this version:}

Isabel D. Alves, Sophie Lecomte. Study of G-Protein Coupled Receptor Signaling in Membrane Environment by Plasmon Waveguide Resonance. Accounts of Chemical Research, 2019, 52 (4), pp.10591067. 10.1021/acs.accounts.9b00007 . hal-02381024

\section{HAL Id: hal-02381024 \\ https://hal.science/hal-02381024}

Submitted on 22 Dec 2020

HAL is a multi-disciplinary open access archive for the deposit and dissemination of scientific research documents, whether they are published or not. The documents may come from teaching and research institutions in France or abroad, or from public or private research centers.
L'archive ouverte pluridisciplinaire HAL, est destinée au dépôt et à la diffusion de documents scientifiques de niveau recherche, publiés ou non, émanant des établissements d'enseignement et de recherche français ou étrangers, des laboratoires publics ou privés. 


\section{Study of G-protein coupled receptor signaling in membrane environment by plasmon waveguide}

\section{resonance}

Isabel D. Alves* and Sophie Lecomte*

CBMN UMR 5248 CNRS, U. of Bordeaux, Bat. B14 allée Geoffroy St. Hilaire, 33600 Pessac, France

KEYWORDS: G-protein coupled receptors, plasmon waveguide resonance, lipid model membrane, ligand-receptor interactions, protein reconstitution 
CONSPECTUS: Here we describe an experimental technique, termed Plasmon Waveguide Resonance (PWR) Spectroscopy that enables the characterization of molecular interactions occurring at the level of anisotropic thin films as lipid membranes and therein inserted or interacting molecules. PWR allows one to characterize such molecular interactions at different levels: 1) acquire binding curves and calculate dissociation constants; 2) obtain kinetic information; 3) obtain information about associated anisotropy changes; 4) get insight about lateral homogeneity. When applied to membrane proteins as the G-protein Coupled Receptor (GPCR) family, both ligand-induced conformational changes of the receptor can be followed as well as interactions with effectors (e.g. G-proteins). Additionally, by either altering the lipid composition in cellular membranes or specifically controlling its composition in the case of lipid model membranes with reconstituted proteins, the role of the lipid environment in receptor activation and signaling can be determined. Additionally, the eventual partition of receptors in different lipid microdomains can be followed. Such information can be obtained with cell membranes fragments expressing the protein of interest and/or lipid model systems where the protein under investigation has been reconstituted. Moreover, PWR can also be applied to directly follow the reconstitution of membrane proteins in lipid model membranes. The measurements are performed directly (no labeling of molecular partners), in real time and with very high sensitivity.

Here we will discuss different aspects on GPCR activation and signaling where PWR brought important information in parallel with other approaches. The utility of PWR is not limited to GPCRs but can be applied to any membrane protein. PWR is also a great tool to characterize the interaction of membrane active molecules with lipids. A brief section is dedicated to such 
applications. To finalize, as PWR is a homemade technology, ongoing instrument developments aiming at breaking current experimental limitations are briefly discussed.

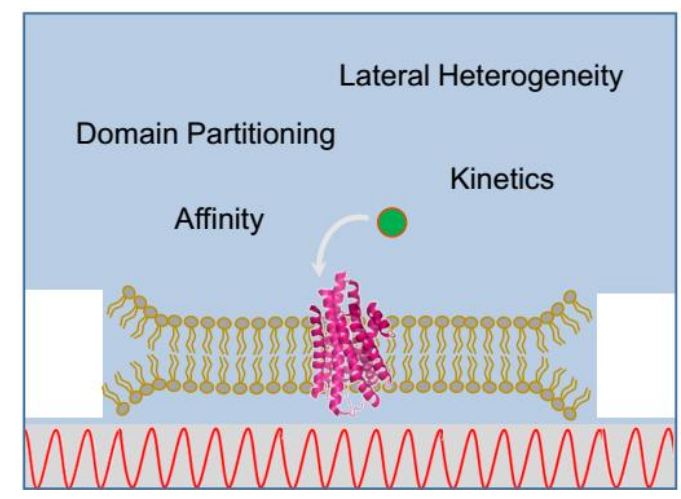

\section{INTRODUCTION}

Molecular interactions play a central role in the regulation of several physiological responses. Those established between membrane receptors and environmental stimuli or via external therapeutic agents play major roles in several cellular processes: signal transduction, nutrient uptake, motility, etc ${ }^{1}$. One important and large class of membrane receptors are GPCRs, 7 transmembrane proteins that sense molecules outside the cell, activate intracellular signal transduction pathways and modulate cellular responses. Since they are activated by a large panoply of extracellular stimuli of varied size and nature such as light, odorants, hormones, and neurotransmitters, these receptors are extremely crucial therapeutic targets ${ }^{2-3}$. Moreover, because they can finely tune cellular responses, they are targets of choice for specific pharmacological treatments. Over the last decades, a huge effort has gone into the identification of molecules that target specific receptors and even subtypes of downstream signalling events, aimed at developing highly-selective treatments. Such development requires a deep understanding of the physico- 
chemical determinants of their behaviour and the interplay between the different actors: ligand, receptor, lipid, effector. Studies on this class of proteins are hampered by the difficulties of working with them due to their inherent properties: hydrophobicity, low natural abundance, difficulty in maintaining their functionality when isolated from their natural lipid environment, among others.

Here we describe a tool, termed PWR, enabling the quantitative characterization of molecular interactions directly (receptor/ligand, receptor/effector) or indirectly (modulation of protein activity by lipids, lipid partitioning) implicated in GPCR activation and signalling ${ }^{4-6}$. PWR allows the characterization of such molecular interactions in terms of affinity $\left(K_{D}\right.$ from $p M$ to $\mathrm{mM}$ ) and kinetics (msec) directly (no labelling needed) and with a very high sensitivity (femtomole). Such studies can be performed both ex-cellulo, that is with cell membrane fragments over expressing the receptor under investigation, and in vitro with the receptor reconstituted in lipid model membranes of controlled lipid composition.

\section{PLASMON WAVEGUIDE RESONANCE METHOD}

While Surface Plasmon Resonance (SPR) is routinely employed in many laboratories to characterize various molecular interactions, supported by the development of specific sensors for the different applications ${ }^{7-10}$, PWR has emerged as a powerful SPR variant for the study of molecular interactions occurring in uniaxially oriented anisotropic thin films as proteolipid membranes $^{5}$. One particular and important difference concerns the sensor design: while SPR uses sensors consisting of a plasmon generating material (usually gold), PWR sensors consist in a metal layer ( $50 \mathrm{~nm}$ of silver is used because it provides sharper resonances than gold) over 
coated by a thick dielectric layer ( $460 \mathrm{~nm}$ of silica) that function as a waveguide. Plasmon and waveguide modes are coupled in PWR measurements ${ }^{4}$. This has important consequences, namely the fact that with SPR only $p$-polarized light (perpendicular to the sensor surface) can be used to create resonances, while in PWR resonances can be obtained with both $p$ - and $s$ - (parallel to the sensor) polarized light. SPR is sensitive to changes in mass occurring upon molecular interactions which allows to obtain affinity constants between the molecular partners. Due to the possibility of PWR to obtain resonances with both $p$ - and $s$-pol, the method becomes sensitive to both changes in mass and anisotropy (see further) that occur as a result of molecular interactions (Fig. 1) ${ }^{4}$. Taking into consideration that both lipids and GPCRs are anisotropic molecules, anisotropy data provides insight about their molecular orientation. This information is crucial for such studies, as explained below. Additional advantages include: 1) higher spectral resolution of PWR compared to SPR supported by the increased electromagnetic field due to the silica. 2) sensitivity of the $p$-pol, $s$-pol and the total internal reflection (TIR) signal to the distance from the sensor surface is different. Indeed, while $s$-pol and $p$-pol decrease exponentially with the distance from the surface (the $s$-pol sensitivity dropping at considerably lower distance than $p$-pol), the TIR signal is constant all the way from the sensor surface to the bulk. This has important consequences in data analysis as the TIR signal can be indicative of non-bound (bulk) material.

3) The dielectric layer serves as mechanical and chemical shield for the metal layer, allowing reactive metals as silver to be used in aqueous environment ${ }^{4}$. PWR sensors are quite resistant and can be reused for months, decreasing running costs.

\section{PWR DATA ANALYSIS}

Different types of PWR data analysis are possible. In any case, important control experiments are necessary to ensure proper data analysis. Most commonly and routinely, to determine the affinity 
of either protein/ligand or lipid/ligand interactions, a plot of ligand concentration (total ligand) versus resonance minimum position obtained at each particular concentration and fit with appropriated equation allows determination of $\mathrm{K}_{\mathrm{D}}$ values (further described below) ${ }^{11}$.

To get further insight into the nature of the changes that occur upon specific molecular interactions, graphical analysis can be applied. The method is based on the fact that PWR spectra are determined by two physical properties of a thin film such as a lipid membrane: an average surface mass density and the spatial distribution of mass within the system that results from the structure of the deposited film ${ }^{12}$. The separation of mass changes from those caused by structure can be obtained by transforming the measured spectral changes (magnitude and direction of the changes in the resonance minimum position obtained for both $p$ - and $s$-pol) from an $(s-p)$ orthogonal coordinate system into one reflecting (mass-structure) (see for details ${ }^{12}$ and applications $^{13}$ ).

The most complete analysis involves spectral fitting. This type of analysis cannot always be applied, exceptions due to the complexity of the system, poor spectra quality, etc. This analysis is supported by the thin-film electromagnetic theory based on Maxwell's equations that provide an analytical relationship between experimental spectral parameters (spectral position, width and depth) and the optical properties of the system (refractive index $n$, extinction coefficient $k$ and thickness $t$ ). As only $n$ and $k$ have different values along the measurement axes: 5 optical parameters must be considered: $n_{p}, n_{s}, k_{p}, k_{s}$ and t. Nonlinear least-square fitting of the experimental to theoretical spectra can be performed to obtain the optical parameters ${ }^{4}$ which can be used to further characterize the system. From the optical parameters, the anisotropy of the refractive index can be calculated to provide information about the molecular orientation and 
ordering. The surface mass density of the system can also be calculated from Lorentz-Lorentz equation (for details see ${ }^{4}$ ) allowing the number of proteins in a lipid membrane to be determined.

\section{PROTOCOLS FOR RECEPTOR IMMOBILISATION IN THE SENSOR}

Plasmon resonance methods require the immobilization of one of the molecules under study on the sensor surface. A large choice of sensor chips with functionalized surfaces has been developed for SPR allowing the target molecule to be attached covalently or non-covalently. While SPR is uncontestably valuable and routinely used to characterize diverse molecular interactions, its application for uniaxially oriented films as lipid bilayers is rather limited. Indeed, since SPR does not provide information about anisotropy or molecular orientation it is unable to ascertain proper membrane formation. Non-ideal lipid membranes (unproperly formed or oriented, with defaults, etc) can compromise results due to surface heterogeneity. Other approaches providing anisotropy information as Dual Polarization Interferometry ${ }^{10}$ or PWR, among others, are thus ideal.

\section{Solid-supported planar membranes}

Solid-supported lipid membranes can be formed by two alternative procedures: 1) by the same principles that govern the spontaneous formation of freely suspended lipid bilayers, a method developed by Mueller and Rudin ${ }^{14}$ to form bilayers across holes in teflon. In PWR studies, such bilayer is anchored to a teflon orifice by an annulus of lipid solution (the plateau Gibbs border) that allows lipid molecules to move into and out of the bilayer in response to protein insertion and/or conformation changes. The protocol has been thoroughly described elsewhere ${ }^{4-5} .2$ ) by fusion of small unilamellar vesicles (SUVs) with the silica surface. Due to their high membrane curvature, upon contact with silica they spontaneously burst to form a lipid membrane ${ }^{15}$. 


\section{Cell membrane fragments}

Two different protocols might be used to immobilize cell membrane fragments: 1) following isolation of membrane fraction from cells (expressing or not the protein of interest) they are incubated with the sensor in presence of $5 \mathrm{mM} \mathrm{CaCl}_{2}$. This protocol was successfully applied to

membrane fragments from mammalian cells in culture and from animal tissue ${ }^{16}$. immobilization of cell fragments on the sensor pre-coated with polylysine (PLL) by electrostatic interaction between sugars in cell membranes and PLL (adapted from Vogel et al ${ }^{17}$ ). It allows cell membrane fragment immobilization from cells in culture (detailed protocols found here ${ }^{18-19}$ ).

\section{STUDY OF GPCR SIGNALING IN RECONSTITUTED LIPID MODEL MEMBRANES}

Despite the difficulties in working with reconstituted systems, they are mandatory to totally control lipid membrane composition and restrain to one single protein component.

\section{GPCR reconstitution in lipid model membranes}

Following GPCR isolation from cellular membranes using detergents, reconstitution in lipid model membranes is necessary to maintain their stability. Two different strategies can be used to follow GPCR reconstitution: 1) the detergent-solubilized receptor is reconstituted in SUVs following different reconstitution procedures ${ }^{20}$ (dilution or detergent removal using dialysis, gelfiltration, hydrophobic absorption using Bio-beads, etc) and the proteolipid membrane is formed by spontaneous proteoliposome fusion with the silica surface; 2) a planar lipid model membrane is formed on the PWR sensor first by one of the two methods presented above, then receptor is then reconstituted in this pre-formed lipid bilayer by detergent dilution below the critical micelle concentration $(\mathrm{cmc})$. While this second procedure is advantageous as it allows one to directly 
monitor GPCR reconstitution while evaluating associated problems (partial or total detergent membrane solubilisation, no protein insertion, protein insertion in non-expected orientation, etc), the first method might be necessary when the second fails (detergent concentration or nature is too harsh for the lipid membrane, unfavorable detergent to protein ratio, sample protein concentration is too low, etc). Most reconstitution procedure require: first separation of reconstituted species from non-reconstituted ones by sucrose gradients or other purification methods, then validation by SDS-PAGE and Western-blot or by biophysical methods (NMR, IR and Raman, EPR, CD, etc), depending among others on the lipid model system. Alternatively, fluorescence microscopy or other imaging methods are used to analyze the reconstituted protein in giant unilamellar vesicles (GUVs), which requires labeled material.

The reconstitution of the chemokine receptor CCR5 into planar lipid membranes has been directly monitored by PWR and rate constants for the process obtained ${ }^{21}$. To do so, a small volume of detergent-solubilized receptor is inserted in the PWR chamber filled with buffer with a pre-formed lipid bilayer. Detergent concentration decreases below the $\mathrm{cmc}$, favoring spontaneous protein insertion in the membrane. Ideally, for the process to proceed well and to avoid unwanted deleterious detergent effect on the lipid membrane (leading to large defaults due to partial membrane solubilisation), the protein is in a detergent with not too low cmc (detergents as dodecylmaltoside are quite deleterious for the membrane) as octylglucoside. Since octylglucoside often fails in keeping most receptor biological activity, an exchange of detergents just before the reconstitution process should be envisaged. Alternatively, if the protein is at considerably high concentration and the addition of a very small volume of detergent-solubilized protein to the PWR chamber for reconstitution has no impact in membrane integrity. Both approaches have been applied for the reconstitution of the chemokine CCR5 receptor and 
showed to be successful ${ }^{21}$. The added value of PWR for this particular application resides on the fact that within the same experiment both the reconstitution and receptor activity, in terms of ligand recognition, can be directly determined with very high sensitivity. Indeed, most approaches do not allow for both aspects to be investigated, following receptor reconstitution, the activity of the protein is measured in yet a diff erent experimental setup with often a tedious and long procedure relying in high quantities of material and labeled proteins. PWR provides a good alternative with the only limitation being that the detergent in which the protein is solubilized must not perturb membrane integrity.

\section{Receptor interaction with ligands and effectors}

Once the receptor has been properly reconstituted in the lipid membrane, its activation by ligand can be monitored and ligand affinity measured. By comparing the data with reported $\mathrm{K}_{\mathrm{D}}$ values receptor activity can be validated, following isolation and reconstitution procedures. To determine ligand binding affinity, ligand is incrementally added to the proteolipid system and resonance spectral changes measured for each concentration. By plotting ligand concentration (total ligand) versus resonance shifts obtained for each concentration when the system reached equilibrium (no spectral changes observed with time; this reflects the R/L complex) and fitting by hyperbolic function that describes classical ligand binding, the dissociation constant for the process can be obtained ${ }^{11}$. Since the majority of GPCRs ligands are quite small and the affinities are very high, the small amounts of low molecular weight ligands do not contribute to mass changes measured by PWR. Therefore, spectral changes observed are mainly attributed to ligand-induced receptor conformational changes. PWR has successfully been applied to monitor ligand binding of several GPCRs. Studies with the hDOR involving a large panoply of ligands 
revealed that different classes of ligands (agonists, antagonists, inverse agonists) lead to very different receptor conformational states ${ }^{11}$.

Besides receptor/ligand interaction, PWR was also applied to study G-protein interaction $\left(1^{\text {st }}\right.$ effector following receptor activation) with receptor, either in the unbound or ligand-bound state $^{22-23}$. Most approaches employed to monitor receptor/G-protein interaction, besides FRET or BRET assays, are rather indirect as they measure further downstream signaling (GTP $\gamma \mathrm{S}$ and cAMP). Moreover, such assays reflect the response of a mixture of G-protein subunits naturally expressed in the cell system used. Pioneer PWR studies of G-protein/receptor interaction reported in 2003 were indeed the first studies able to directly measure such interaction and associated affinity. The affinity of the G-protein to the receptor/ligand complex and that of the $\mathrm{GTP} \gamma \mathrm{S}$ for the ligand/receptor/G-protein was separately determined showing no direct correlation between the efficacy of a receptor to recognize a G-protein and to induce GTP/GDP exchange ${ }^{23}$. It also showed that while both unoccupied, agonist and antagonist-occupied hDOR are all able to bind to G-proteins, only the agonist-bound receptor induces GTP/GDP exchange. Moreover, by investigating the interaction of different $\mathrm{hDOR}$ ligand-bound states with different G-protein subunits $\left(\mathrm{G}_{\mathrm{i} \alpha 1}, \mathrm{G}_{\mathrm{i} \alpha 2}, \mathrm{G}_{\mathrm{i} \alpha 3}, \mathrm{G}_{\mathrm{o} \alpha}\right)$, the selectivity of such interactions was evidenced. Indeed, certain ligands have a high degree of selectivity in terms of the G-protein they activate while others don' $\mathrm{t}^{22-23}$. This has important implications for agonist-direct trafficking and rational drug design whereby ligands could be designed that target specific pathways, avoiding known detrimental side effects of certain drugs.

\section{Lipid modulation of receptor activity}


Since GPCRs are almost completely immersed in the lipid membrane, the composition of such must impact their structure and function ${ }^{24}$. Lipids can exert their action in a "specific" manner whereby bound lipids act as chemical partners, or in a "bulk" manner by modulating the physical lipid properties (fluidity, curvature, surface tension, etc) and thereby affecting protein function. Cholesterol is highly abundant representing more than $20 \%$ of lipids in mammalian cell membranes ${ }^{25}$. Its presence modulates the fluidity of the lipid membrane and also the formation of lipid domains (lipid rafts that are enriched in cholesterol and sphingomyelin, SM) consisting of highly ordered and packed lipid regions that co-exist with less ordered ones mainly composed of unsaturated phospholipids ${ }^{26}$. Studies have indicated that cholesterol is required for the entry of HIV virions into cells expressing the co-receptor CCR5 and/or CXCR4 (co-receptors implicated in HIV-infection), and that cholesterol depletion inhibits HIV entry ${ }^{27}$. Furthermore, cholesterol depletion leads to failure of CCR5 to inhibit cAMP activity ${ }^{28}$. As most studies performed relied in the use of $\beta$-cyclodextrin to deplete cholesterol from the membrane, a molecule associated with several $\operatorname{artifacts}^{29}$, more direct studies are needed. Ligand binding to CCR5 in model lipid membranes containing or not cholesterol was investigated by PWR. The data indicates an enhancement of the binding affinity of maraviroc, one of the first commercialized anti HIV agents, to membranes containing cholesterol, and that independently of the presence of sphingomyelin, therefore independent of the presence or lipid-ordered domains ${ }^{21}$.

In another study the effect of the membrane curvature on the light-activation of rhodopsin was investigated. For such, rhodopsin was reconstituted in lipid membranes composed of lamellar lipids (phosphatidylcholine) in absence or presence of non-lamellar lipids (phosphatidylethanolamine, PE) that form negative membrane curvature. By PWR the $\mathrm{pH}$ dependent transition from the photointermediate metarhodopsin I to metarhodopsin II was 
followed and the pKa of the transition determined. An increase of the pka of 1 unity was observed when rhodopsin was reconstituted in PE enriched membranes. Moreover, the affinity of the light-activated rhodopsin for transducin (the rhodopsin G-protein) was also impacted by the lipid membrane composition, with a 25 -fold increase in affinity when PE was present ${ }^{30}$. While the impact of $\mathrm{PE}$ in the meta I/meta II transition was determined by flash-photolysis experiments $^{31-32}$, it was the first time that a study suggested that lipid impact could propagate beyond the receptor level (at rhodopsin-transducin interaction).

\section{Receptor partition in lipid domains}

Lipid membranes are not laterally homogeneous assemblages but possess nano or microdomains due to the spontaneous segregation of certain types of lipids in the plane of the membrane ${ }^{33-34}$. Indeed, lipid microdomains form spontaneously in mixtures of SM and unsaturated phospholipids (as POPC or DOPC), in both presence and absence of cholesterol ${ }^{33}$. Microdomains could provide more specificity and diversity in the signaling cascade by providing compartmentalization of the different components that are involved or by modulating the intrinsic activities of proteins therein located ${ }^{35}$. Therefore, it is very much pertinent to understand how GPCRs partition between such domains, and more importantly how such partition is impacted by receptor occupancy and bound-state. In the beginning of 2000, we ask ourselves whether by PWR measurements, one could discriminate between the lipid-ordered and disordered domains and eventually follow receptor partition between such domains. PWR measurements of two-component lipid membranes, known to laterally separate to form microdomains (namely, POPC/SM at 1/1 mol/mol), performed with the classic PWR sensor (50 
$\mathrm{nm}$ of silver overcoated by $460 \mathrm{~nm}$ of $\mathrm{SiO}_{2}$ ) resulted in wider PWR spectra but rather symmetric (non-published data). This lead us to think that this sensor did not possess high enough resolution to discriminate lipid microdomains. Another sensor was developed with a more elaborated composition that resulted in much sharper spectra ${ }^{36}$. With this sensor, spectra obtained for the binary lipid mixtures could be ascribed to a superposition of two resonances corresponding to microdomains consisting of POPC- and SM-rich phases coexisting within a single bilayer. Indeed, spectra deconvolution and simulation, allowed to attribute part of the spectra to the PCrich region (at lower resonance angle) and the other part to the SM-rich region (at higher resonance angle). Optical parameters were obtained for each of these regions, demonstrating that the PC-rich region is less thick and less packed than the SM-rich region. This data is in very good agreement with AFM literature that demonstrate these two lipid domains to have about $1 \mathrm{~nm}$ thickness difference. In such binary lipid membranes, the partitioning and functioning of the hDOR in the two domains was investigated as well as the impact of the ligand on receptor partitioning. The study demonstrate that the partitioning was highly dependent on the type of ligand bound to the receptor: in the unbound-state the receptor preferentially partitions in the PCrich domain, while when activated by agonist it partitions preferentially in the SM rich domain (with two fold-greater propensity for this microdomain). Moreover, the addition of ligand to an unbound receptor leads to its trafficking from the PC-rich to the SM-rich domain. This study revealed that receptor partitioning impacts the interaction with the G-protein, a 30-fold higher affinity was observed for its interaction with the receptor present in the SM-rich domain ${ }^{37}$. While these studies did not go far enough to determine the membrane property that was at the origin of such partitioning and impact in first receptor signaling events, one could suppose that the lipid environment impacts the receptor conformational state. The preferential partition of the agonist- 
bound receptor into the thicker SM-rich domain could be triggered by hydrophobic matching, as the activated receptor-state should be more elongated than the non-activated one.

\section{STUDY OF GPCR SIGNALING IN CELL FRAGMENTS}

Certain aspects on GPCR activation and signaling can alternatively be performed on the natural GPCR environment, namely the cells overexpressing the receptor of interest. As explained above, protocols have been established and validated for the immobilization of cell fragments on the PWR sensor. With this approach the affinity of ligands to the chemokine receptors CXCR3 and the ligand-induced receptor conformational changes have been evaluated. The CXCR3 receptor regulates several biological functions and plays important roles in angiogenesis, inflammation and cancer ${ }^{38-39}$. One peculiarity of this receptor is that there are two distinct isoforms that have antagonistic effects in cancer development and angiogenesis: the CXCR3-A isoform is implicated in cell proliferation, migration and cell survival and the CXCR3-B isoform (that differs from the A form by an additional 52 residues in the N-term) displays angiostatic, anti-proliferative and apoptotic functions ${ }^{40}$. Extended literature supports the idea that the activation of these two CXCR3 isoforms leads to distinct biological behaviors and clinical outcomes, which could be explained by very early changes in the two signaling pathways. With the objective of understanding the origin of such diverging responses in the signaling mediated by the two receptor isoforms, PWR experiments were performed to measure the affinity of ligands to each receptor isoform and associated conformational changes. The studies revealed that the affinity of an agonist to the two receptors was substantially different and most interesting that the conformational changes associated were completely different ${ }^{18}$. This could explain why 
different signaling cascades are activated by ligand-binding to the two receptors, as recently reported $^{41}$. PWR along with calcium-flux experiments were able to define a very narrow window of a receptor antagonist concentration that specifically inhibited the agonist binding and activation of the CXCR3-A isoform, while having negligible effect on the CXCR3-B isoform. This could have important pathological perspectives as the CXCR3-A isoform is ultimately triggering tumor progression, its specific inhibition relative to the B isoform (triggering tumor regression) is desirable. Moreover, this reveals the very high fine-tuning of GPCRs.

\section{PWR AS A TOOL TO INVESTIGATE THE INTERACTION OF MEMBRANE ACTIVE MOLECULES WITH LIPIDS}

Besides GPCRs, our research group is focused on the understanding of the dynamics and the mechanisms of interaction between membrane active peptides and biological or biomimetic membranes.

We employ biophysical approaches as polarized ATR-FTIR, PMIRRAS to evaluate structural changes of peptides and proteins and lipid organization. PWR allows a very complementary description by bringing the kinetic aspect and determination of binding affinities. PWR has been applied to investigate the interaction of cell penetrating peptides with both model membranes and cell membrane fragments from cells expressing or not certain glycosaminoglycans allowing both the lipid and sugar role to be determined ${ }^{42}$.

For 10 years, a strong interest of the team has been on the relation between morphology and structure of amyloid peptides, and toxicity expressed by their deleterious effects on the biological membranes ${ }^{43}$. Our first study was to develop in the yeast Saccharomyces cerevisiae a model of amyloid toxicity based on toxic mutants of the HET-s prion of Podospora anserina. Our collaborators have created 40 mutants of varying toxicity. A reported highlight was that toxic 
species, deleterious for the membranes are small objects (oligomers), structured in anti-parallel $\beta$-sheets while fibers structured in parallel $\beta$-sheet, had in turn no disruptive effect on membrane models ${ }^{43}$. In this project, PWR made it possible not only to establish the kinetic interaction constants of the HET-s and mutant peptides with different model membranes, but above all to highlight the presence of different forms or sizes of aggregates interacting with these membranes ${ }^{5}$. The addition of the toxic peptide to the lipid membrane lead to the appearance of additional resonances in the s-pol that was attributed to heterogeneity in peptide assembly with the presence of regions (domains) possessing different mass and organization. Taking into account the ability of amyloid peptides to oligomerize and to form fibers and plates the multiple resonances seen can correspond to different oligomeric states of the peptides.

Similarly, more recent work on the peptide $A \beta_{1-42}$ involved in Alzheimer's disease by PWR allowed us to establish that the least toxic species (monomers) interact with the membrane but that the oligomeric species induce a reorganization of the lipids of the membrane. The interaction was decomposed into two phases with two different kinetic constants between the anchorage of the oligomers on the membrane and the induced reorganization of the lipids ${ }^{44}$.

\section{CONCLUSIONS}

PWR provides an interesting technique to characterize molecular interactions occurring in thin anisotropic films as lipid membranes and herein inserted membrane proteins (as GPCRs) or interacting molecules as membrane active peptides. For studies with model lipid membranes, other systems besides supported bilayers can be used (polymer cushioned, partially suspended, hybrid, etc) depending on the system under investigations. Moreover, immobilisation strategies 
(implicating chemical modification of the surface) allowing controlled and homogeneous orientation of the target molecule in the sensor surface can be applied.

As any other method, PWR has also its limitations. One limitation regards the distinction of the spectral contribution of each molecular partner in complex systems. For example, when a ligand binds and activates a receptor, with the current PWR setup and by measuring at a single wavelength it is impossible to determine if the spectral changes reflect conformational changes of the receptor alone or lipid reorganization contribution. To circumvent this limitation, there are two possibilities: 1) to fluorescently label one of the partners and to measure the PWR signal at two wavelengths. Both the anisotropy of the refractive index and that of the extinction coefficient (see for details ${ }^{4,}{ }^{45}$ ) are determined allowing one to distinguish each molecular contribution $\left.^{46} .2\right)$ Another possibility that we are currently starting to explore concerns the expansion of PWR measurements from the visible into the IR-region. Since lipids and proteins have inherent and specific IR absorption bands, by measuring at appropriate wavelengths, specific molecular information can be obtained.

Another limitation concerns the lack of information regarding lipid membrane electrochemical properties. To follow changes in membrane electrochemical properties upon pore formation or opening of a channel could be highly interesting. Such development is important because it would: help establish the mechanism of action of membrane active peptides (e.g. pore formation); allow protein ionophore properties to be followed, allow the role of membrane potential in receptor activity to be determined; to follow electrochemically the activity of certain proteins as redox enzymes. While this has been pioneered by Tollin, that included an electrode in the sensor design PWR spectra quality is yet poor (depth loss) ${ }^{47}$. The design of a new sensor with 
improved PWR signals, while keeping optimal electrochemical potential, is currently under development.

\section{FIGURES}

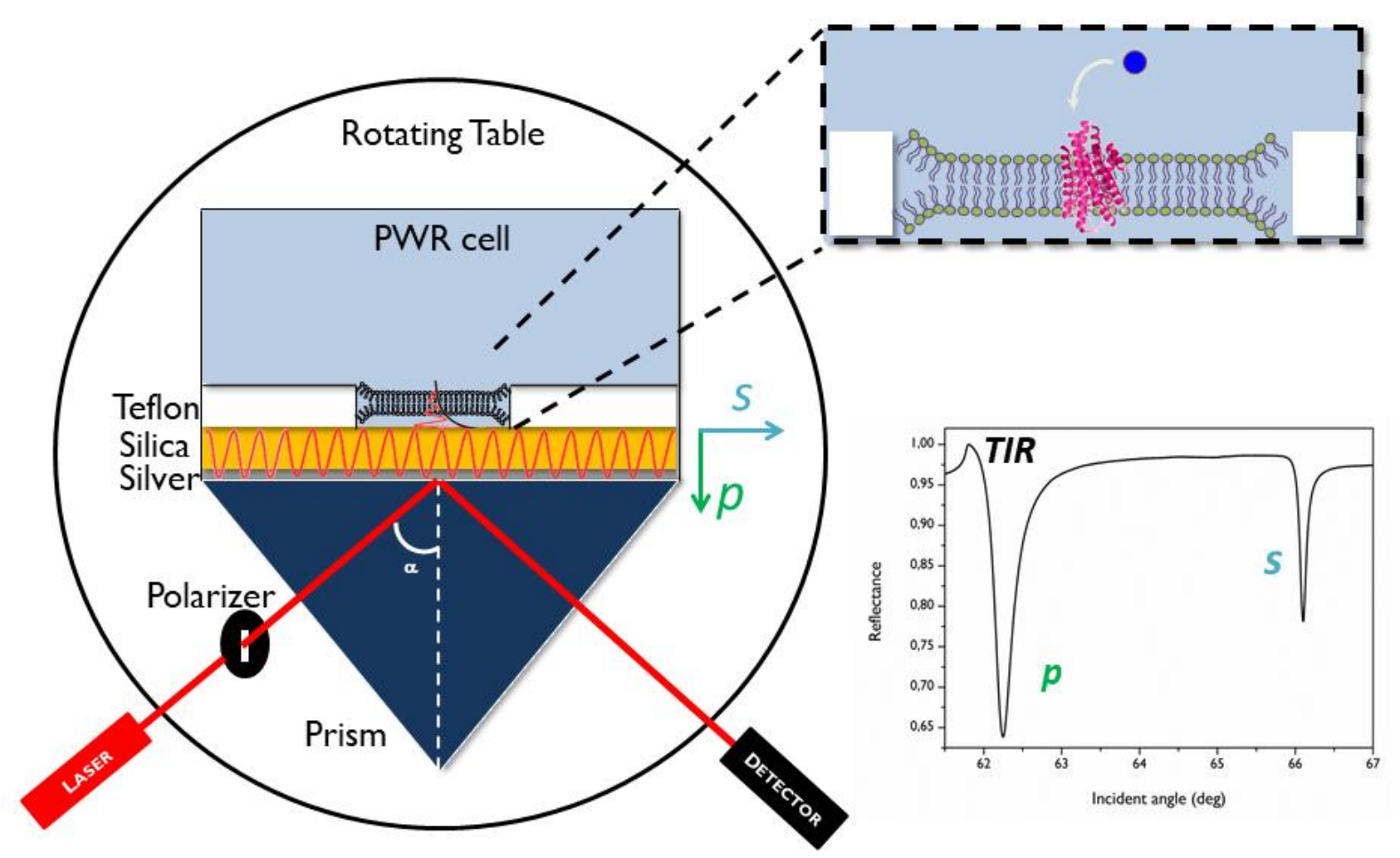

Figure 1. PWR setup. On the left the optical and mechanical components, the incident polarized light beam (a continuous $\mathrm{He}-\mathrm{Ne}$ laser at $632.8 \mathrm{~nm}$ ) and the rotating table allows steps of 1 millidegree. The sensor consists of a right angle BK7 prism coated with a $50 \mathrm{~nm}$ layer of silver overcoated by $460 \mathrm{~nm}$ of silica. $S$ - (parallel) and $p$-(perpendicular) polarized light are defined relative to the sensor surface. On the right top is a detailed view of the PWR cell sample 
with the lipid bilayer, embedded receptor and ligand or effector binding. On the right bottom is a typical PWR spectra showing the total internal reflection angle (TIR), $p$ - and $s$-resonances.

\section{AUTHOR INFORMATION}

\section{Corresponding Author}

*Corresponding authors: Isabel D. Alves (‥alves@,cbmn.u-bordeaux.fr) and Sophie Lecomte (s.lecomte@,cbmn.u-bordeaux.fr). Address: CBMN UMR 5248 CNRS, U. of Bordeaux, Bat B14, allée Geoffroy St. Hilaire, 33600 Pessac, France

\section{Author Contributions}

The manuscript was written through contributions of both authors. All authors have given approval to the final version of the manuscript.

\section{BIOGRAPHIES}

Dr. Sophie Lecomte did her undergraduate studies in Chemistry at the University Pierre and Marie Curie (UPMC) in Paris (France) and received her PhD in 1995 from that university. After postdoctoral studies at the University of Strathclyde (Glasgow, UK) and the Max Planck Institute for Radiation Chemistry (Melheim an der Ruhr, Germany), she joined the Laboratory of Dynamics, Interactions and Reactivity in the UPMC as CNRS research scientist (1998). In 2007, she moved to the Institute of Chemistry and Biology of Membranes and Nanoobjects in Bordeaux (France). Her current research focuses on self-assembling processes of amyloid peptides implicated in Alzheimer's disease and their interaction with membranes. 
Dr. Isabel Alves did her undergraduate studies in Biochemistry in the University of Algarve (Portugal). In 2004 she received her $\mathrm{PhD}$ in Biochemistry from the University of Arizona (Tucson, USA). Following a short postdoctoral work in the Laboratory of Biomolecules at the University Pierre and Marie Curie in Paris (France) she was appointed CNRS research scientist in this same laboratory (2006). In 2010 she moved to the Institute of Chemistry and Biology of Membranes and Nanoobjects in Bordeaux (France). Her current research focus in the mechanisms of action of membrane active peptides and the role of lipids in the activation and signaling of GPCRs.

\section{REFERENCES}

1. Uings, I. J.; Farrow, S. N., Cell receptors and cell signalling. Mol Pathol 2000, 53 (6), $295-9$.

2. Kobilka, B., The structural basis of G-protein-coupled receptor signaling (Nobel Lecture). Angew Chem Int Ed Engl 2013, 52 (25), 6380-8.

3. Lagerstrom, M. C.; Schioth, H. B., Structural diversity of G protein-coupled receptors and significance for drug discovery. Nature reviews. Drug discovery 2008, 7 (4), 339-57.

4. Salamon, Z., et al., Coupled plasmon-waveguide resonators: a new spectroscopic tool for probing proteolipid film structure and properties. Biophysical journal 1997, 73 (5), 2791-7.

5. Harte, E., et al., Probing the kinetics of lipid membrane formation and the interaction of a nontoxic and a toxic amyloid with plasmon waveguide resonance. Chemical Communications 2014, 50 (32), 4168-4171.

6. Salamon, Z., et al., Chapter 6. Plasmon resonance methods in membrane protein biology applications to GPCR signaling. Methods Enzymol 2009, 461, 123-46.

7. Homola, J., Surface plasmon resonance sensors for detection of chemical and biological species. Chem Rev 2008, 108 (2), 462-93.

8. Karlsson, R., SPR for molecular interaction analysis: a review of emerging application areas. Journal of molecular recognition : JMR 2004, 17 (3), 151-61.

9. Nguyen, H. H., et al., Surface plasmon resonance: a versatile technique for biosensor applications. Sensors (Basel) 2015, 15 (5), 10481-510.

10. Lee, T. H., et al., Exploring Molecular-Biomembrane Interactions with Surface Plasmon Resonance and Dual Polarization Interferometry Technology: Expanding the Spotlight onto Biomembrane Structure. Chem Rev 2018, 118 (11), 5392-5487.

11. Alves, I. D., et al., Different structural states of the proteolipid membrane are produced by ligand binding to the human delta-opioid receptor as shown by plasmon-waveguide resonance spectroscopy. Molecular pharmacology 2004, 65 (5), 1248-57.

12. Salamon, Z.; Tollin, G., Graphical analysis of mass and anisotropy changes observed by plasmon-waveguide resonance spectroscopy can provide useful insights into membrane protein function. Biophysical journal 2004, 86 (4), 2508-16. 
13. Alves, I. D., et al., The interaction of cell-penetrating peptides with lipid model systems and subsequent lipid reorganization: thermodynamic and structural characterization. Journal of peptide science 2009, 15 (3), 200-9.

14. Mueller, P.; Rudin, D. O., Resting and action potentials in experimental bimolecular lipid membranes. $J$ Theor Biol 1968, 18 (2), 222-58.

15. E. Reimhult, F. H., B. Kasemo, Intact vesicle adsorption and supported biomembrane formation from vesicle sin solution: influence of surface chemistry, vesicle size, temeprature, and osmotic pressure. Langmuir 2002, 19, 1681-1691.

16. Salamon, Z., et al., Plasmon-waveguide resonance studies of ligand binding to integral proteins in membrane fragments derived from bacterial and mammalian cells. Analytical biochemistry 2009, 387 (1), 95-101.

17. Perez, J. B., et al., Monitoring the diffusion of single heterotrimeric G proteins in supported cell-membrane sheets reveals their partitioning into microdomains. Journal of molecular biology 2006, 363 (5), 918-30.

18. Boye, K., et al., Ligand activation induces different conformational changes in CXCR3 receptor isoforms as evidenced by plasmon waveguide resonance (PWR). Sci Rep 2017, 7 (1), 10703.

19. Boye, K., et al., The role of CXCR3/LRP1 cross-talk in the invasion of primary brain tumors. Nat Commun 2017, 8 (1), 1571.

20. Goddard, A. D., et al., Reconstitution of membrane proteins: a GPCR as an example. Methods Enzymol 2015, 556, 405-24.

21. Calmet, P., et al., Real time monitoring of membrane GPCR reconstitution by plasmon waveguide resonance: on the role of lipids. Sci Rep 2016, 6, 36181.

22. Alves, I. D., et al., Selectivity, cooperativity, and reciprocity in the interactions between the delta-opioid receptor, its ligands, and G-proteins. Journal of biological chemistry 2004, 279 (43), 44673-82.

23. Alves, I. D., et al., Direct observation of G-protein binding to the human delta-opioid receptor using plasmon-waveguide resonance spectroscopy. Journal of biological chemistry 2003, 278 (49), 48890-7.

24. Lee, A. G., Biological membranes: the importance of molecular detail. Trends in biochemical sciences 2011, 36 (9), 493-500.

25. van Meer, G., et al., Membrane lipids: where they are and how they behave. Nature reviews. Molecular cell biology 2008, 9 (2), 112-24.

26. de Meyer, F.; Smit, B., Effect of cholesterol on the structure of a phospholipid bilayer. Proc. Nat. Acad. Sci. 2009, 106 (10), 3654-8.

27. Ablan, S., et al., The role of cholesterol and sphingolipids in chemokine receptor function and HIV-1 envelope glycoprotein-mediated fusion. Virol $J$ 2006, 3, 104.

28. Cardaba, C. M., et al., CCR5 internalisation and signalling have different dependence on membrane lipid raft integrity. Cell Signal 2008, 20 (9), 1687-94.

29. Plazzo, A. P., et al., Uptake of a fluorescent methyl-beta-cyclodextrin via clathrindependent endocytosis. Chemistry and physics of lipids 2012, 165 (5), 505-11.

30. Alves, I. D., et al., Phosphatidylethanolamine enhances rhodopsin photoactivation and transducin binding in a solid supported lipid bilayer as determined using plasmon-waveguide resonance spectroscopy. Biophysical journal 2005, 88 (1), 198-210.

31. Botelho, A. V., et al., Conformational energetics of rhodopsin modulated by nonlamellarforming lipids. Biochemistry 2002, 41 (20), 6354-68. 
32. Botelho, A. V., et al., Curvature and hydrophobic forces drive oligomerization and modulate activity of rhodopsin in membranes. Biophysical journal 2006, 91 (12), 4464-77. 33. Simons, K.; Vaz, W. L., Model systems, lipid rafts, and cell membranes. Annu Rev Biophys Biomol Struct 2004, 33, 269-95.

34. Dietrich, C., et al., Lipid rafts reconstituted in model membranes. Biophysical journal 2001, 80 (3), 1417-28.

35. Insel, P. A., et al., Compartmentation of G-protein-coupled receptors and their signalling components in lipid rafts and caveolae. Biochem Soc Trans 2005, 33 (Pt 5), 1131-4.

36. Salamon, Z., et al., Plasmon-waveguide resonance studies of lateral segregation of lipids and proteins into microdomains (rafts) in solid-supported bilayers. Journal of biological chemistry 2005, 280 (12), 11175-84.

37. Alves, I. D., et al., Ligand modulation of lateral segregation of a G-protein-coupled receptor into lipid microdomains in sphingomyelin/phosphatidylcholine solid-supported bilayers. Biochemistry 2005, 44 (25), 9168-9178.

38. Belperio, J. A., et al., CXC chemokines in angiogenesis. J Leukoc Biol 2000, 68 (1), 1-8.

39. Groom, J. R.; Luster, A. D., CXCR3 in T cell function. Exp Cell Res 2011, 317 (5), 620-

31.

40. Billottet, C., et al., CXCR3, a double-edged sword in tumor progression and angiogenesis. Biochimica et biophysica acta 2013, 1836 (2), 287-95.

41. Smith, J. S., et al., C-X-C Motif Chemokine Receptor 3 Splice Variants Differentially Activate Beta-Arrestins to Regulate Downstream Signaling Pathways. Molecular pharmacology 2017, 92 (2), 136-150.

42. Alves, I. D., et al., Cell biology meets biophysics to unveil the different mechanisms of penetratin internalization in cells. Biochimica et biophysica acta 2010, 1798 (12), 2231-9.

43. Berthelot, K., et al., What does make an amyloid toxic: morphology, structure or interaction with membrane? Biochimie 2013, 95 (1), 12-9.

44. Henry, S., et al., Interaction of Abeta(1-42) amyloids with lipids promotes "off-pathway" oligomerization and membrane damage. Biomacromolecules 2015, 16 (3), 944-50.

45. Alves, I. D., Introduction to Plasmonics Advances and Applications.

46. Salamon, Z.; Tollin, G., Optical anisotropy in lipid bilayer membranes: coupled plasmonwaveguide resonance measurements of molecular orientation, polarizability, and shape.

Biophysical journal 2001, 80 (3), 1557-67.

47. Salamon, Z., et al., Plasmon-waveguide resonance and impedance spectroscopy studies of the interaction between penetratin and supported lipid bilayer membranes. Biophysical journal 2003, 84 (3), 1796-807. 